\title{
Approach of Reliability Approximation with Extent of Error for a Resistor under Weibull Setup
}

\author{
Sadananda Nayak \\ Ramakrishna Mission Vidyabhavan, Paschim Midnapore, \\ West Bengal, India \\ sadanandastat@gmail.com \\ Dilip Roy \\ Centre for Management Studies. \\ The University of Burdwan, Golapbag, Burdwan-713104 \\ dr.diliproy@gmail.com
}

Received 28 September 2014

Accepted 3 March 2015

\begin{abstract}
Analytical determination of reliability of a complex system is item dependent and setup dependent and a very difficult task. In this paper we propose reliability approximation based on reliability bounds for one such engineering item - a Resistor with independent components having the Weibull distribution. Earlier approach for reliability approximation was discretization. We provide a different approach for reliability approximation so that one not only gets a clear idea about the extent of error but also can adjust reliability in terms of distributional parameters. This reliability approximation having the Weibull distribution can be of practical use and importance as reliability approximation is developed in terms of distributional parameters.
\end{abstract}

Keywords: Stress-Strength Model, System Reliability, Weibull distribution, Reliability bound, Reliability approximation, Extent of error. 


\section{Notations}

$$
\begin{aligned}
& S_{x}(X)=\text { Survival function (sf) of a random variable } \mathrm{X} \text { evaluated at the time point } \mathrm{X} \text {. } \\
& F_{x}(X) \quad=\text { Corresponding cumulative distribution }(\mathrm{df}) \text { function } . \\
& \mathrm{W}(\lambda, \alpha) \quad=\text { Weibull distribution with scale parameter } \lambda \text { and shape parameter } \alpha \text {. } \\
& \mathrm{E}(\mathrm{X}) \quad=\text { Expectation on random variable } \mathrm{X} \text {. } \\
& \mathrm{R} \quad=\text { System reliability. } \\
& U\left(\lambda, \lambda_{V}, \lambda_{R_{1}}, \lambda_{R_{2}}\right)=\text { Reliability upper bound under the Weibull setup. } \\
& L\left(\lambda, \lambda_{V}, \lambda_{R_{1}}, \lambda_{R_{2}}\right)=\text { Reliability lower bound under the Weibull setup. } \\
& R_{\text {approx }} \quad=\text { Reliability approximation when } \mathrm{m}>1, \mathrm{n}>0 \text { and } \alpha>2 \text {. } \\
& R_{\text {approx }}^{* *} \quad=\text { Reliability approximation when } \mathrm{m}<1, \mathrm{n}>0 \text { and } \alpha>2 \text {. } \\
& R * * *{ }_{\text {approx }} \quad=\text { Reliability approximation when } \mathrm{m}<1, \mathrm{n}<0 \text { and } \alpha>2 \text {. } \\
& \text { Error* } \quad=\text { Extent of error when } \mathrm{m}>1, \mathrm{n}>0 \text {, and } \alpha>2 \text {. } \\
& \text { Error** } \quad=\text { Extent of error when } \mathrm{m}<1, \mathrm{n}<0 \text { and } \alpha>2 \text {. } \\
& \text { Error*** } \quad=\text { Extent of error when } \mathrm{m}<1, \mathrm{n}>0 \text { and } \alpha>2 \text {. }
\end{aligned}
$$

\section{Introduction}

In the context of reliability, stress indicates (Kapur and Lamberson [4]) any agency that tends to induce failure. On the other hand Strength indicates any agency resisting failure. Under the stress-strength model, reliability is defined as the probability that strength is greater than stress.

Knowing the distribution function of stress and strength, (Gertsbakh [3]) the reliability of the system i.e. the probability that the strength $\mathrm{S}$ is greater than the stress $\mathrm{Y}$ can be obtained using an integral equation.

Ordinary transformation techniques due to Parzen [5] can be used to determine the system reliability when stress and strength distribution are directly known. However such analytical approaches virtually fail when the stress variable is made up of multiple random factors.

\section{Problem in determination}

For many engineering items, the stress or strength variables are themselves functions of multiple stochastic factors and problem arises in determination of reliability. Now-a-days there are three independent lines of attack. First line of attack is due to Taylor's series expansion. Second line of attack follows from range approximation method which is given by Roy and Dasgupta [11]. In third approach, continuous setup is replaced by a closely approximated discrete setup given by Roy [8].

\section{Earlier works}

D'Errico and Zaino [1] have used Taguchi's [14] concept of experimental design and they presented discretization approach for approximating the behavior of complex system. Later English et al [2] extended the some approach by increasing the number of discrete point. They compared discrete approximation with simulated values. But their study was of course limited to normal setup. Xie and Lai [6] have studied approximation of system reliability using one step conditioning. Xue and Yang [15] have established a stress-strength inference reliability model with strength degradation under the assumptions that stress-strength are statistically independent. They have also presented simple formulas for estimating upper and lower 
bounds for stress-strength reliability. The concept of discrete concentration of Roy [7] was used by Roy and Dasgupta [12] for presenting discretizing procedure. Roy [9 - 10] have examined in details discrete normal and discrete Rayleigh Distribution in this process. Roy and Dasgupta [13] have proposed evaluation of reliability of complex systems by means of discretizing approach under the Weibull setup.

But discrete approximation for any engineering item cannot adjust in terms of the parameter of the distribution. Reliability bounds on the other hand are functions of distributional parameters. Keeping these issues in mind, we have approximated reliability based on reliability bounds.

\section{Reliability bounds}

For the purpose of our proposed work, we have considered a well-known engineering item for approximating the reliability based on reliability bounds. The choice of our engineering item is power dissipated by a resistor.

A resistor is made up of two resistances $R_{1}$ and $R_{2}$ connected in parallel. The power dissipated from the resistor is described by the following functional form:

$Y=V^{2}\left(\frac{1}{R_{1}}+\frac{1}{R_{2}}\right)$, where $\mathrm{Y}$ is the shear stress, $\mathrm{V}$ is the voltage across each resistor, and $R_{1}$ and $R_{2}$ are two resistances.

Let us evaluate the reliability approximation based on reliability bounds for a resistor having the Weibull distributions for $R_{1}, R_{2}$ and $\mathrm{V}$.

\section{Reliability bounds under the Weibull life distribution}

Let us assume that component random variables $R_{1}, R_{2}$ and $\mathrm{V}$ are independent having the following parametric set ups. $\mathrm{V} \sim \mathrm{W}\left(\lambda_{V}, \alpha\right), R_{1} \sim \mathrm{W}\left(\lambda_{R_{1}}, \alpha\right)$, and $R_{2} \sim W\left(\lambda_{R_{2}}, \alpha\right)$. Let the built- in- strength $\mathrm{S}$ be such that $\mathrm{S}$ follows yet another $\mathrm{W}(\lambda, \alpha)$.Under these assumption, we try to determine the bounds on system reliability R. For this, we consider the following lemmas.

Lemma 1: Let $\mathrm{X}$ be nonnegative function of a r.v. $\mathrm{X}$, then for every $\mathrm{t}, \mathrm{P}(\mathrm{X} \geq t) \leq \frac{E(X)}{t}$

Lemma 2: Let $\mathrm{X}$ be nonnegative function of a r.v. $\mathrm{X}$, then for every $\mathrm{t}, \mathrm{P}(\mathrm{X} \leq t) \geq 1-\frac{E(X)}{t}$

Theorem 1: Upper bound $U\left(\lambda, \lambda_{V}, \lambda_{R_{1}}, \lambda_{R_{2}}\right)$, for the system reliability $\mathrm{R}$, for a Resistor having the Weibull distribution is given by

$$
U\left(\lambda, \lambda_{V}, \lambda_{R_{1}}, \lambda_{R_{2}}\right)=\operatorname{Min}\left[\left(\frac{\lambda^{2}}{\lambda}\right)^{\frac{1}{\alpha}} \frac{\Gamma\left(\frac{1}{\alpha}+1\right) \Gamma\left(1-\frac{2}{\alpha}\right)\left\{\Gamma\left(\frac{1}{2 \alpha}+1\right)\right\}^{2}}{2\left(\lambda_{R_{1}} \lambda_{R_{2}}\right)^{\frac{1}{2 \alpha}}}, 1\right], \text { for } \alpha>2 .
$$

Proof: From the definition of reliability

$$
\begin{aligned}
\mathrm{R} & =\mathrm{P}(\mathrm{S} \geq Y) \\
& =\mathrm{P}\left(\mathrm{S} \geq V^{2}\left(\frac{1}{R_{1}}+\frac{1}{R_{2}}\right)\right)
\end{aligned}
$$




$$
\begin{aligned}
& =\mathrm{P}(\mathrm{S} \geq \mathrm{L}), \text { where } \mathrm{L}=V^{2}\left(\frac{1}{R_{1}}+\frac{1}{R_{2}}\right) \\
& \leq E\left(\frac{E(S)}{L}\right), \text { applying lemma1. } \\
& =E(S) E\left(\frac{1}{L}\right) \\
& =E(S) E\left(\frac{1}{V^{2}\left(\frac{1}{R_{1}}+\frac{1}{R_{2}}\right)}\right) \\
& =E(S) E\left(\frac{1}{V^{2}}\right) E\left(\frac{1}{\frac{1}{R_{1}}+\frac{1}{R_{2}}}\right)
\end{aligned}
$$

Now using the relation between G.M. and H.M., (1) reduce to

$$
\begin{aligned}
\mathrm{R} & \leq E(S) E\left(\frac{1}{V^{2}}\right) E\left(\frac{1}{2} \sqrt{R_{1}} \sqrt{R_{2}}\right) \\
& =\frac{E(S) E\left(\frac{1}{V^{2}}\right) E\left(\sqrt{R_{1}}\right) E\left(\sqrt{R_{2}}\right)}{2} \\
& =\left(\frac{\lambda^{2}{ }_{V}}{\lambda}\right)^{\frac{1}{\alpha}} \frac{\Gamma\left(\frac{1}{\alpha}+1\right) \Gamma\left(1-\frac{2}{\alpha}\right)\left\{\Gamma\left(\frac{1}{2 \alpha}+1\right)\right\}^{2}}{2\left(\lambda_{R_{1}} \lambda_{R_{2}}\right)^{\frac{1}{2 \alpha}}}=\mathrm{m}, \text { say, }
\end{aligned}
$$

Therefore, under the Weibull distribution, upper bound for the system reliability $\mathrm{R}$, for a Resistor is

$$
U\left(\lambda, \lambda_{V}, \lambda_{R_{1}}, \lambda_{R_{2}}\right)=\left(\frac{\lambda^{2}{ }_{V}}{\lambda}\right)^{\frac{1}{\alpha}} \frac{\Gamma\left(\frac{1}{\alpha}+1\right) \Gamma\left(1-\frac{2}{\alpha}\right)\left\{\Gamma\left(\frac{1}{2 \alpha}+1\right)\right\}^{2}}{2\left(\lambda_{R_{1}} \lambda_{R_{2}}\right)^{\frac{1}{2 \alpha}}} \text {, for } \alpha>2 .
$$

If upper bound crosses 1 then it should be truncated at 1 . Hence

$$
U\left(\lambda, \lambda_{V}, \lambda_{R_{1}}, \lambda_{R_{2}}\right)=\operatorname{Min}\left[\left(\frac{\lambda^{2}{ }_{V}}{\lambda}\right)^{\frac{1}{\alpha}} \frac{\Gamma\left(\frac{1}{\alpha}+1\right) \Gamma\left(1-\frac{2}{\alpha}\right)\left\{\Gamma\left(\frac{1}{2 \alpha}+1\right)\right\}^{2}}{2\left(\lambda_{R_{1}} \lambda_{R_{2}}\right)^{\frac{1}{2 \alpha}}}, 1\right], \text { for } \alpha>2
$$

It completes the proof of theorem 1.

Theorem 2: Lower bound $L\left(\lambda, \lambda_{V}, \lambda_{R_{1}}, \lambda_{R_{2}}\right)$, for the system reliability $\mathrm{R}$, for a Resistor having the Weibull distribution is given by

$$
L\left(\lambda, \lambda_{V}, \lambda_{R_{1}}, \lambda_{R_{2}}\right)=\operatorname{Max}\left[0,1-\sqrt{2} \Gamma\left(1-\frac{1}{2 \alpha}\right)\left(\frac{\sqrt{\lambda}}{\lambda_{V}}\right)^{\frac{1}{\alpha}}\left(\lambda_{R_{1}}+\lambda_{R_{2}}\right)^{\frac{1}{2 \alpha}}\right], \text { for } \alpha>\frac{1}{2} .
$$


Proof: From the definition of reliability

$$
\begin{aligned}
\mathrm{R} & =\mathrm{P}(\mathrm{S} \geq Y) \\
& =\mathrm{P}\left(\mathrm{S} \geq V^{2}\left(\frac{1}{R_{1}}+\frac{1}{R_{2}}\right)\right) \\
& =\mathrm{P}\left(V \leq \sqrt{\frac{R_{2} S R_{1}}{R_{2}+R_{1}}}\right) \\
& =\mathrm{P}\left(V \leq L^{\prime}\right), \text { where } L^{\prime}=\sqrt{\frac{R_{2} S R_{1}}{R_{2}+R_{1}}} \\
& \geq 1-E\left(\frac{E(V)}{L^{\prime}}\right), \text { applying lemma2. } \\
& =1-E(V) E\left(\frac{1}{L^{\prime}}\right) \\
& =1-E(V) E\left(\frac{1}{\sqrt{S}}\right) E\left(\sqrt{\frac{R_{1}+R_{2}}{R_{1} R_{2}}}\right) \\
\mathrm{R} & \geq 1-E(V) E\left(\frac{1}{\sqrt{S}}\right) \frac{\sqrt{2}}{\left.E\left(\sqrt{M i n\left(R_{1} R_{2}\right.}\right)\right)} \\
& \left.=1-\sqrt{2} \Gamma\left(1-\frac{1}{2 \alpha}\right)\left(\frac{\sqrt{\lambda}}{\lambda_{V}}\right)^{\frac{1}{\alpha}\left(\lambda_{R_{1}}\right.}+\lambda_{R_{2}}\right)^{\frac{1}{2 \alpha}}=\mathrm{n}, \text { say, }
\end{aligned}
$$

Therefore, under the Weibull distribution, lower bound for the system reliability $\mathrm{R}$, for a Resistor is

$$
L\left(\lambda, \lambda_{V}, \lambda_{R_{1}}, \lambda_{R_{2}}\right)=\operatorname{Max}\left[0,1-\sqrt{2} \Gamma\left(1-\frac{1}{2 \alpha}\right)\left(\frac{\sqrt{\lambda}}{\lambda_{V}}\right)^{\frac{1}{\alpha}}\left(\lambda_{R_{1}}+\lambda_{R_{2}}\right)^{\frac{1}{2 \alpha}}\right] \text {, for } \alpha>\frac{1}{2} .
$$

Because the lower bound cannot fall below zero.

It completes the proof of theorem2.

\section{Reliability approximation and error term}

We propose the mean of the upper and lower bound as the approximate value of the system reliability and half of the absolute deviation between the two bounds as the extent of error. Let us discuss the three cases to derive reliability approximation and error terms. This reliability approximation and corresponding error terms can be expressed in terms of distributional parameters.

\section{Case1: Reliability approximation and error term when $m>1, n>0$ and $\alpha>2$}

Using the theorem 1 and theorem 2 , we propose to approximate the system reliability as

$$
\begin{aligned}
R_{\text {approx }} & =\frac{[\operatorname{Min}(m, 1)+\operatorname{Max}(0, n)]}{2} \\
& =1-\left\{\Gamma\left(1-\frac{1}{2 \alpha}\right)\left(\frac{\sqrt{\lambda}}{\lambda_{V}}\right)^{\frac{1}{\alpha}}\left(\lambda_{R_{1}}+\lambda_{R_{2}}\right)^{\frac{1}{2 \alpha}}\right\} / \sqrt{2}
\end{aligned}
$$


This is a function of distributional parameters. Therefore, we can adjust it in respect of distributional parameters.

Extent of error is given by

$$
\begin{aligned}
\text { Error* } & \leq \frac{[\operatorname{Min}(m, 1)-\operatorname{Max}(0, n)]}{2} \\
& =\left\{\Gamma\left(1-\frac{1}{2 \alpha}\right)\left(\frac{\sqrt{\lambda}}{\lambda_{V}}\right)^{\frac{1}{\alpha}}\left(\lambda_{R_{1}}+\lambda_{R_{2}}\right)^{\frac{1}{2 \alpha}}\right\} / \sqrt{2}
\end{aligned}
$$

Case2: Reliability approximation and error term when $\mathbf{m}<\mathbf{1 ,} \mathbf{n}>\mathbf{0}$ and $\alpha>\mathbf{2}$

Under this assumption, using theorem1 and theorem2, we suggest to approximate the system reliability as

$$
\begin{aligned}
& R *{ }_{\text {approx }}= \frac{[\operatorname{Min}(m, 1)+\operatorname{Max}(0, n)]}{2} \\
&=\left[\left(\frac{\lambda^{2} V}{\lambda}\right)^{\frac{1}{\alpha}} \frac{\Gamma\left(\frac{1}{\alpha}+1\right) \Gamma\left(1-\frac{2}{\alpha}\right)\left\{\Gamma\left(\frac{1}{2 \alpha}+1\right)\right\}^{2}}{2\left(\lambda_{R_{1}} \lambda_{R_{2}}\right)^{\frac{1}{2 \alpha}}}\right. \\
&\left.+1-\sqrt{2} \Gamma\left(1-\frac{1}{2 \alpha}\right)\left(\frac{\sqrt{\lambda}}{\lambda_{V}}\right)^{\frac{1}{\alpha}}\left(\lambda_{R_{1}}+\lambda_{R_{2}}\right)^{\frac{1}{2 \alpha}}\right] / 2
\end{aligned}
$$

Extent of error is given by

$$
\begin{aligned}
& \text { Error** } \leq \\
& \qquad \begin{aligned}
=\left[\left(\frac{\lambda^{2} V}{\lambda}\right)^{\frac{1}{\alpha}} \frac{\Gamma\left(\frac{1}{\alpha}+1\right) \Gamma\left(1-\frac{2}{\alpha}\right)\left\{\Gamma\left(\frac{1}{2 \alpha}+1\right)\right\}^{2}}{2}\right. \\
2\left(\lambda_{R_{1}} \lambda_{R_{2}}\right)^{\frac{1}{2 \alpha}} \\
\left.-1+\sqrt{2} \Gamma\left(1-\frac{1}{2 \alpha}\right)\left(\frac{\sqrt{\lambda}}{\lambda_{V}}\right)^{\frac{1}{\alpha}}\left(\lambda_{R_{1}}+\lambda_{R_{2}}\right)^{\frac{1}{2 \alpha}}\right] / 2
\end{aligned}
\end{aligned}
$$

Case3: Reliability approximation and error term when $\mathbf{m}<\mathbf{1 ,} \mathbf{n}<\mathbf{0}$ and $\alpha>2$

Under this assumption, using theorem1 and theorem2, we propose to approximate the system reliability as

$$
\begin{aligned}
R * * *_{\text {approx }} & =\frac{[\operatorname{Min}(m, 1)+\operatorname{Max}(0, n)]}{2} \\
& =\left(\frac{\lambda^{2}{ }_{V}}{\lambda}\right)^{\frac{1}{\alpha}} \frac{\Gamma\left(\frac{1}{\alpha}+1\right) \Gamma\left(1-\frac{2}{\alpha}\right)\left\{\Gamma\left(\frac{1}{2 \alpha}+1\right)\right\}^{2}}{4\left(\lambda_{R_{1}} \lambda_{R_{2}}\right)^{\frac{1}{2 \alpha}}}
\end{aligned}
$$

Extent of error can be given as

$$
\begin{aligned}
\text { Error*** } & \leq \frac{[\operatorname{Min}(m, 1)-\operatorname{Max}(0, n)]}{2} \\
& =\left(\frac{\lambda^{2}{ }_{V}}{\lambda}\right)^{\frac{1}{\alpha}} \frac{\Gamma\left(\frac{1}{\alpha}+1\right) \Gamma\left(1-\frac{2}{\alpha}\right)\left\{\Gamma\left(\frac{1}{2 \alpha}+1\right)\right\}^{2}}{4\left(\lambda_{R_{1}} \lambda_{R_{2}}\right)^{\frac{1}{2 \alpha}}}
\end{aligned}
$$




\section{Numerical study}

By varying the mean strength parameter $\lambda$, we make a numerical study of reliability approximation along with the extent of error. The specific choices of distributional parameters are $\lambda_{V}=25, \lambda_{R_{1}}=4, \lambda_{R_{2}}=5$. The corresponding reliability approximations and error terms have been provided in Table1. It may be observed that error term sharply decreases as reliability increases.

\section{An application of the proposed work}

Unlike simulation method or discrete approximation method, design parameters can be adjusted under this method. Hence it can be of practical use during the early stages of product design. As reliability bounds are function of design parameters, are very important for product planning when neither the discrete approximation nor the actual values are available. There are cases where discrete approximations are extremely weak. For example, under the exponential setup, where lack of memory property holds, the discretization approach does not offer close approximate values. In that situation, one may depend on reliability bounds.

\section{References}

[1] D'Errico, J. R. and Zaino, N.A. Jr (1988). Statistical tolerancing using a modification of Taguchi's method, Technometrics, 30, 397-405.

[2] English, J.R. Sargent, T. and Landers, T.L. (1996). A discretizing approach for stress $\backslash$ strength analysis, IEEE Trans. Reliability, 45, 84-89.

[3] Gertsbakh, I. B. (1989). Statistical Reliability Theory, Marcel Dekker Inc., New York and Basel.

[4] Kapur, K.C. and Lamberson, L.R. (1976). Reliability in Engineering Design, John Wiley and Sons.

[5] Parzen, E. (1960). Modern probability theory and its application, John Wiley and Sons.

[6] Xie, M and Lai,C.D. (1998). Reliability bounds via conditional inequalities, Journal of applied probability, 35,104-114.

[7] Roy, D. (1993). Reliability measures in the discrete bivariate setup and related characterization results for a bivariate geometric distribution, J. Multivariate Analysis, 46, 362-373.

[8] Roy, D. (2002). Discretization of continuous distributions, Calcutta Statistical Association Bulletin, 52, 297-313.

[9] Roy, D. (2003). The discrete normal distribution, Communications in Statistics (Theory and Methods) 32, 1871-1883.

[10] Roy, D. (2004). Discrete Rayleigh Distribution, IEEE: Trans. Reliability, 53, 255-260.

[11] Roy, D. and Dasgupta, T. (2000). A continuous approximation for evaluating reliability of complex systems under stress - strength model, Communications in statistics (Simulation and Computation), 29, 811-821.

[12] Roy, D. and Dasgupta, T. (2001). A discretizing approach for evaluating reliability of complex systems under stress - strength model, IEEE Trans. Reliability, 50, 145-150.

[13] Roy, D. and Dasgupta, T. (2002). Evaluation of reliability of complex systems by means of a discretizing approach: Weibull setup, International Journal for Quality and Reliability Management, 19, 792-801.

[14] Taguchi, G. (1978). Performance Analysis Design, International journal of production Research, $16,521-530$.

[15] Xue, J, and Yang, K. (1997). Upper and lower bounds of stress-strength inference reliability with random strength degradation, 46, 142-145. 


\section{Appendix}

Table 1: Study of reliability approximation and error terms under the Weibull distribution for shape parameter $\alpha=3$.

\begin{tabular}{|c|c|c|}
\hline Strength parameter $\lambda$ & Reliability approximation & Extent of error \\
\hline .0009 & .877654 & .122346 \\
\hline .0008 & .880032 & .119968 \\
\hline .0007 & .882672 & .117328 \\
\hline .0006 & .885648 & .114352 \\
\hline .0005 & .889071 & .110929 \\
\hline .0004 & .893119 & .106881 \\
\hline .0003 & .898119 & .101877 \\
\hline .00025 & .901173 & .098828 \\
\hline .00020 & .904780 & .095220 \\
\hline .00015 & .909238 & .090763 \\
\hline .00012 & .912551 & .087449 \\
\hline .00010 & .915168 & .084832 \\
\hline .00005 & .924423 & .075577 \\
\hline .00004 & .9272183 & .072818 \\
\hline .00003 & .930591 & .069409 \\
\hline .00002 & .935126 & .064874 \\
\hline .00001 & .942204 & .057796 \\
\hline .000007 & .945539 & .054460 \\
\hline .000003 & .952712 & .047288 \\
\hline .0000015 & .957871 & .042129 \\
\hline .0000007 & .962896 & .037104 \\
\hline .0000003 & .967783 & .032217 \\
\hline .0000001 & .973173 & .026827 \\
\hline .00000002 & .979484 & .020516 \\
\hline .00000001 & .981722 & .018278 \\
\hline .000000001 & .987548 & .012453 \\
\hline .0000000001 & .991516 & .008484 \\
\hline .000000000001 & .996062 & .003938 \\
\hline
\end{tabular}

